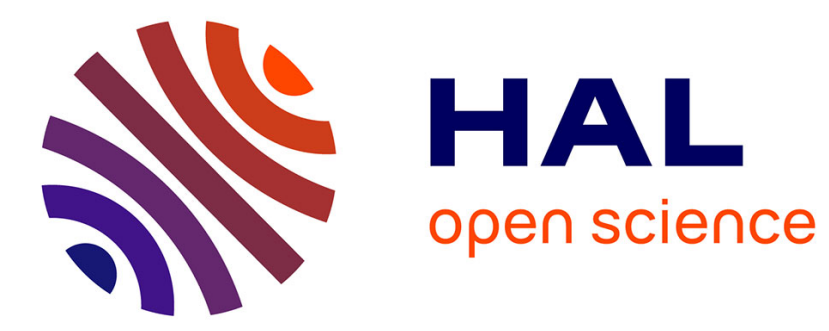

\title{
Natural Element Meshless Simulation of Injection Processes Involving Short Fiber Suspensions
}

Miguel Angel Martínez, Elías Cueto, Manuel Doblaré, Francisco Chinesta

\section{To cite this version:}

Miguel Angel Martínez, Elías Cueto, Manuel Doblaré, Francisco Chinesta. Natural Element Meshless Simulation of Injection Processes Involving Short Fiber Suspensions. Journal of Non-Newtonian Fluid Mechanics, 2003, 115 (1), pp.51-78. 10.1016/S0377-0257(03)00171-X . hal-00020745

\section{HAL Id: hal-00020745 https://hal.science/hal-00020745}

Submitted on 1 Mar 2018

HAL is a multi-disciplinary open access archive for the deposit and dissemination of scientific research documents, whether they are published or not. The documents may come from teaching and research institutions in France or abroad, or from public or private research centers.
L'archive ouverte pluridisciplinaire HAL, est destinée au dépôt et à la diffusion de documents scientifiques de niveau recherche, publiés ou non, émanant des établissements d'enseignement et de recherche français ou étrangers, des laboratoires publics ou privés. 


\title{
Natural element meshless simulation of flows involving short fiber suspensions
}

\author{
M.A. Martínez ${ }^{\text {a }}$, E. Cueto ${ }^{\text {a }}$, M. Doblaré ${ }^{\mathrm{a}, *}$, F. Chinesta $^{\mathrm{b}}$ \\ a Departamento de Ingeniería Mecánica, División de Mecánica Estructural, \\ Universidad de Zaragoza, María de Luna 3, 50015 Zaragoza, Spain \\ b Laboratoire de Mécanique des Systèmes et des Procédés (CNRS-ENSAM-ESEM), \\ 151 Boulevard de l'Hôpital, F-75013 Paris, France
}

\begin{abstract}
Numerical modeling of non-Newtonian flows typically involves the coupling between the equations of motion characterized by an elliptic character, and the fluid constitutive equation, which is an advection equation linked to the fluid history. Thus, the numerical modeling of short fiber suspensions flows requires a description of the microstructural evolution (fiber orientation) which affects the flow kinematics and that is itself governed by this kinematics (coupled problem). Some industrial flows involve moving or free boundaries (injection, extrusion, ... ). Lagrangian descriptions allow an accurate description of the flow front tracking as well as an accurate integration of transport equations along the flow trajectories. However, Lagrangian techniques in the context of finite elements have the important drawback of requiring frequent remeshing in order to avoid large elements distortions. The natural element method (NEM) has the capabilities of Lagrangian models to describe the flow front tracking as well as to treat the convection terms related to the fiber orientation equation without the mesh quality requirement characteristics of the standard finite elements method.
\end{abstract}

Keywords: Meshless methods; Natural element method; $\alpha$-Shapes; Fixed mesh Eulerian simulations; Volume-of-fluid method; Advection equation; Non-Newtonian fluids; Short fibers molten composites

\section{Introduction}

As a consequence of the increasing use of composite materials, there has been much work on constitutive equations and computational mechanics for short fibers composites. Since these materials are generally made of a matrix and fibers reinforcement, the mechanical properties of the conformed pieces depend greatly on the fibers orientation in the solid material. However, it turns out that this orientation

* Corresponding author. Tel.: +34-976-76-19-13; fax: +34-976-76-18-61.

E-mail addresses: mdoblare@posta.unizar.es (M. Doblaré), francisco.chinesta@paris.ensam.fr (F. Chinesta). 
is determined by the forming process, so that it is interesting to develop mathematical models describing the flow during this conforming process and to develop specific numerical strategies to solve the resulting equations.

Mechanical modeling of short fibers suspensions flows is usually achieved in the framework of dilute or semi-dilute suspensions of non-spherical particles in a Newtonian fluid. The resulting system of equations involves the coupling of an elliptic problem with an advection problem related to the fluid history. The elliptic problem is associated with the equations of motion whereas the advection equation describes the time evolution of the anisotropic viscosity tensor (fiber orientation). The second problem presents two difficulties: it is non-linear and hyperbolic.

Coupled models take into account both the dependence of the kinematics with the fiber orientation and the orientation induced by the flow kinematics. Usually, the coupled models are solved by means of a fixed point strategy. In this case, at each iteration, the flow kinematics results from the solution of motion and mass conservation equations, assuming the fiber orientation field at the previous iteration. From the kinematics just computed, the fiber orientation is updated solving the advection equation governing its evolution. Advection equations can be integrated by using any accurate numerical technique for hyperbolic equations: the method of characteristics, SUPG or discontinuous finite element techniques, discontinuous finite volumes, ... [1-6]. Coupled models solving simultaneously the flow kinematics and the fiber orientation (fully coupled models) are rare in literature. The main difficulty in using fully coupled models is the different character of the model equations, which requires specific numerical techniques.

The simulation of flows involving moving or free boundaries introduces specific difficulties related to the flow front treatment [7]. A first possibility to describe the fluid volume evolution is the use of a fixed mesh strategy. In that case, the fluid volume updating is carried out from a control volume technique or by using a volume-of-fluid (VOF) technique, which introduces a new variable (the fluid presence function) whose evolution is governed, as described in Section 3, by a linear advection equation. Some of these techniques solve the flow kinematics exclusively in the fluid domain, whereas other ones operate in the whole domain imposing a pseudo-behavior in the empty region. The use of this kind of techniques (fixed mesh strategies) induces additional difficulties in the flow front treatment, due to the fact that usual fixed mesh discretization techniques update the fluid properties from their values at the previous time step. Thus, when an element starts its filling process, the variables related to the fluid, such as the temperature, the fiber orientation, ... are not defined in the empty elements, even though initial values are required to start the evolution process. Moreover, in all cases, the position and shape of the flow front is more or less uncertain, because in practice, during the filling simulation a great number of partially-filled elements appear. To improve the flow front location, some alternatives exist, as for example, the level set method, but its use is far to be trivial.

The consideration of a moving mesh strategy (as used for example in the Lagrangian finite element formulations) allows to get a good evaluation of the fluid domain evolution, although some precautions must be taken into account in the flow front tracking: confluent flow fronts, interaction of the flow front with the domain boundary, .... The advection equations related to the fluid history can be accurately integrated using the method of characteristics along the nodal trajectories. However, as it is well known in the context of the Lagrangian finite element method, the mesh becomes too distorted in few iterations to guarantee an accurate field interpolation in the mesh elements. In order to alleviate the remeshing constraint some meshless methods have been proposed [8]. However, usual meshless techniques do not define a nodal interpolation, and in consequence important difficulties are found in the imposition of the essential boundary conditions. The natural element method (NEM), a novel meshless method, has the 
property of nodal interpolation, whose accuracy does not depend on the regularity of nodal distribution, i.e. there is not geometrical restriction in the relative position of the nodes. Thus, if the NEM is used in the discretization of the variational formulation of motion and mass conservation equations, the nodal position can be updated from the velocity field of the fluid, at the same time that advection equations are integrated using the method of characteristics. Even in the case of very irregular nodal distributions, when the solution can be interpolated by using the approximation functional basis, no remeshing is required. Nevertheless, the introduction or elimination of some nodes is an easy task.

This paper does not pretend to simulate real forming process (which will require a deeper work). Its main aim is to show some interesting and promising capabilities of an updated Lagrangian technique using a meshless approximation of both the trial and the test functions in the discretization of the variational formulation related to a short fiber suspension flow. In our opinion, the application of this technique for simulating injection processes involving very complex geometries (as usually encountered in industrial applications) will require a deeper work to adapt (adjust) the nodal density during the simulation (without taking into account their relative position due to the meshless character of the method). For this purpose efficient error indicators are required. First nodal density adaptation tentatives were proposed by Cueto [9] and other possibilities will be published shortly. On the other hand, we can affirm that it is already possible simulating accurately free boundary problems (as encountered in extrusion or spinning processes, among many other) using the updated Lagrangian meshless strategy described in this paper. In this field, and of course in our opinion, the meshless character of our approach has interesting and appealing properties that allow an accurate description of these free or moving boundaries without any geometrical restriction in the nodal position (which is not the case when an updated finite element strategy is used). However, we would like to emphasize that this work does not pretend to indulge in a polemic on mesh based procedures against meshless techniques.

This paper is structured in four main sections. In the first one, the mechanical model governing the flow of an anisotropic suspension will be introduced, as well as the volume of fluid strategy to describe the fluid volume evolution when a fixed mesh of the whole domain is considered. The second and the third ones concern the numerical modeling of that flow using (i) the volume of fluid method to compute the fluid volume evolution in a fixed mesh framework, and (ii) a moving mesh technique based in a remeshing-free technique (the NEM). The filling process of a cavity is simulated in the last section. The main particularity of this numerical example is that when the filling process starts, the flow is not affected by the mold walls, and in consequence it consists actually in an extruded flow.

\section{Mechanical modeling}

The flow model of a short fibers suspension is defined by the following equations [10-14]:

- The balance of momentum equations, without inertia and mass terms

$\operatorname{Div} \underline{\underline{\sigma}}=\underline{0}$,

where $\underline{\sigma}$ is the stress tensor.

- The incompressibility condition

$\operatorname{Div} \underline{v}=0$,

where $\underline{v}$ represents the velocity field. 
- The constitutive equation, with a quadratic closure relation for the fourth-order orientation tensor and other simplifying assumptions [15], results

$$
\underline{\underline{\sigma}}=-p \underline{\underline{I}}+2 \mu\left\{\underline{\underline{D}}+N_{p} \operatorname{Tr}(\underline{\underline{a}} \underline{\underline{D}}) \underline{\underline{a}}\right\}
$$

where $p$ denotes the pressure, $\underline{\underline{I}}$ the unit tensor, $\mu$ the equivalent suspension viscosity, $\underline{\underline{D}}$ the strain rate tensor, $N_{p}$ a scalar parameter depending on both the fiber concentration and the fiber aspect ratio, and $\underline{\underline{a}}$ is the second-order orientation tensor defined by

$$
\underline{\underline{a}}=\oint \underline{\rho} \otimes \underline{\rho} \Psi(\underline{\rho}) \mathrm{d} \underline{\rho}
$$

where $\underline{\rho}$ is the unit vector defining the fiber axis direction, and $\Psi(\underline{\rho})$ is the orientation distribution function, verifying the normality condition

$$
\oint \Psi(\underline{\rho}) \mathrm{d} \underline{\rho}=1 \text {. }
$$

If $\Psi(\rho)=\delta(\rho-\hat{\rho})$, with $\delta(\cdot)$ the Dirac's function, all the orientation probability is concentrated in the direction defined by $\underline{\hat{\rho}}$, and the corresponding orientation tensor results $\underline{\underline{a}}=\hat{\underline{\rho}} \otimes \underline{\hat{\rho}}$, i.e. $a_{i j}=\hat{\rho}_{i} \hat{\rho}_{j}$. In the planar case, the isotropic orientation is defined by the uniform distribution function

$$
\Psi(\underline{\rho})=\frac{1}{2 \pi} .
$$

The orientation tensor related to a planar isotropic orientation distribution is then

$$
\underline{\underline{a}}=\frac{1}{2} \underline{\underline{I}} \text {. }
$$

From a physical point of view, we can consider that the eigenvalues of the second-order orientation tensor $(\underline{a})$ represent the probability of finding the fibers in the direction of the corresponding eigenvectors.

For a sake of simplicity only planar orientations will be considered in this work, which is not very appropriate from a physical point of view when 3D flows are considered (in this case, fibers can rotate in the out-of-plane direction). The numerical examples described in this paper concern 2D flows and planar orientation distributions. However, any of these facts limits the validity of the numerical approach here proposed.

- With a quadratic closure relation [16] the orientation equation is expressed as

$$
\frac{\mathrm{d} \underline{\underline{a}}}{\mathrm{~d} t}=\underline{\underline{\Omega}} \underline{\underline{a}}-\underline{\underline{a}} \underline{\underline{\Omega}}+k(\underline{\underline{D}} \underline{\underline{a}}+\underline{\underline{a}} \underline{\underline{D}}-2 \operatorname{Tr}(\underline{\underline{a}} \underline{\underline{D}}) \underline{\underline{a}})-D_{r}\left(\underline{\underline{a}}-\frac{\underline{\underline{I}}}{2}\right),
$$

where $\underline{\underline{a}}$ satisfies

$$
\left\{\begin{array}{l}
\underline{\underline{a}}=\underline{\underline{a}}^{t} \\
\operatorname{Tr}(\underline{\underline{a}})=1
\end{array}\right.
$$

$\underline{D}$ and $\underline{\underline{\Omega}}$ are the symmetric and skew-symmetric components of Grad $\underline{v}, k$ is a constant that depends on the fiber aspect ratio $r$ (fiber length to fiber diameter ratio): $k=\left(r^{2}-1\right) /\left(r^{2}+1\right)$, and $D_{r}$ is a diffusion coefficient taking into account the fiber-fiber interactions. 
The quadratic closure relation is exact as long as the fibers are locally perfectly aligned. If we consider other closure relations (linear and hybrid) [16] or the natural one [17], the orientation equation changes, but its hyperbolic character remains unchanged. Thus, all the numerical procedures proposed in this paper can also be applied when other closure relations are considered. Effectively, in some cases the problem solution can depend strongly on the closure relation considered, which will require to make special attention for simulating real industrial processes [7,18].

The flow model is defined in the volume occupied by the fluid at time $t, \Omega_{\mathrm{f}}(t)$. On its boundary, $\Gamma_{\mathrm{f}}(t) \equiv \partial \Omega_{\mathrm{f}}(t)$ either the velocity or the traction are imposed:

$$
\underline{v}\left(\underline{x} \in \Gamma_{1}\right)=\underline{v}_{\mathrm{g}},
$$

and

$$
\underline{\underline{\sigma}} \underline{\underline{n}}\left(\underline{x} \in \Gamma_{2}\right)=\underline{F}_{\mathrm{g}},
$$

with $\Gamma_{1} \cup \Gamma_{2}=\Gamma_{\mathrm{f}}(t), \Gamma_{1} \cap \Gamma_{2}=\emptyset$, and where $\underline{n}(\underline{x})$ is the unit outwards vector, defined on the boundary at the point $\underline{x}$. The inflow boundary will be denoted by $\Gamma^{-}$:

$$
\Gamma^{-}=\left\{\underline{x} \in \Gamma_{1}, \underline{v}(\underline{x}) \cdot \underline{n}(\underline{x})<0\right\} .
$$

The flow front will be represented by $\Gamma_{\mathrm{ff}}, \Gamma_{\mathrm{ff}} \subset \Gamma_{2}$ where in general a null traction is prescribed, i.e. $\underline{F}_{\mathrm{g}}\left(\underline{x} \in \Gamma_{\mathrm{ff}}\right)=\underline{0}$.

As the orientation Eq. (8) has an hyperbolic character, its integration only requires an orientation boundary condition on the inflow boundary

$$
\underline{\underline{a}}\left(\underline{x} \in \Gamma^{-}\right)=\underline{a}_{0} .
$$

As at the time $t=0$, we consider the whole domain $\Omega$ empty, the initial condition corresponds with the boundary condition given by Eq. (13). At time $t$, only a part of the whole domain $\Omega$ is occupied by the fluid. The empty region will be noted by $\Omega_{\mathrm{e}}(t)\left(\Omega_{\mathrm{f}}(t) \cup \Omega_{\mathrm{e}}(t)=\Omega\right)$. If we denote by $\Gamma_{\mathrm{e}}(t)$ the boundary of $\Omega_{\mathrm{e}}(t)$, the flow front can be also defined by $\Gamma_{\mathrm{ff}}=\Gamma_{\mathrm{f}}(t) \cap \Gamma_{\mathrm{e}}(t)$. From now on, $\Gamma \equiv \partial \Omega$ denotes the boundary of $\Omega$.

\section{Related works: fixed mesh modeling of filling processes}

In the simulation of the injection process, we consider firstly a fully Eulerian description combined with an explicit strategy which proceeds solving the flow kinematics for a given fluid domain, in which the fiber orientation is assumed known. Now, with the flow kinematics just computed we can update the fluid domain as well as the fiber orientation in the new fluid domain. The simulation process finishes when the mold domain is fully filled, i.e. when $\Omega_{\mathrm{f}}=\Omega$.

This explicit strategy allows to compute an accurate solution of the equations of motion (Eqs. (1)-(3)) using a standard velocity-pressure mixed finite element formulation. The hyperbolic character of advection equations governing the fluid domain and the fibers orientation evolutions, requires specific numerical techniques for its discretization, as for example, the discontinuous finite element method, to solve these advection equations (Eqs. (8) and (15)). Many possibilities exist combining explicit or implicit methods with first or higher-order discretization schemes. 
As just indicated, the flow model is defined in the part of the whole domain (injection mold) $\Omega$ occupied by the fluid at each time $t, \Omega_{\mathrm{f}}(t)$. In order to update the fluid domain we are going to introduce the fluid presence function, $I(\underline{x}, t)$. This function takes a unit value in the fluid region and it is zero in the empty domain:

$$
I(\underline{x}, t)= \begin{cases}1 & \text { if } \quad \underline{x} \in \Omega_{\mathrm{f}}(t) \\ 0 & \text { if } \quad \underline{x} \in \Omega_{\mathrm{e}}(t) .\end{cases}
$$

The evolution of this function is given by the following scalar and linear advection equation

$$
\frac{\partial I}{\partial t}+\underline{v} \cdot \operatorname{Grad} I=0
$$

which is defined in the whole domain. The fluid presence function must verify a boundary condition on the mold inlet (inflow boundary)

$$
I\left(\underline{x} \in \Gamma^{-}, t\right)=1,
$$

as well as an initial condition. If we assume the mold empty at the beginning of the injection process, the initial condition results

$$
I(\underline{x}, t=0)=0 .
$$

The discretization of the equations of motion is carried out by means of a standard mixed finite element technique, using an enriched $P_{1}^{+}\left(P_{1}+\right.$ "bubble") $-C^{0}$ approximation in the velocity interpolation and a linear $P_{1}-C^{0}$ approximation in the pressure interpolation. This functional approximation verifies the LBB condition [19]. In order to extend the variational formulation of the flow equations defined in $\Omega_{\mathrm{f}}(t)$ to the whole domain $\Omega$, we impose a pseudo-behavior in the empty volume, defined by $\underline{v}=\underline{0}$ and $p=0$ [20]. Combining both, the flow and the pseudo-behavior variational formulations, we can define the following problem.

Find $\underline{v} \in\left(H^{1}(\Omega)\right)^{3}$ and $p \in L^{2}(\Omega)$ verifying the essential boundary conditions $\underline{v}\left(\underline{x} \in \Gamma^{-}\right)=\underline{v}_{\mathrm{g}}$ and $\underline{v}\left(\underline{x} \in \Gamma-\Gamma^{-}\right)=\underline{0}$, such that

$$
\begin{aligned}
& \int_{\Omega} f(I) \underline{\underline{\sigma}}: \underline{D}^{*} \mathrm{~d} \Omega+\int_{\Omega} \alpha_{v}(1-f(I)) \underline{v} \cdot \underline{v}^{*} \mathrm{~d} \Omega=0, \\
& \int_{\Omega} f(I) \operatorname{Div} \underline{v} p^{*} \mathrm{~d} \Omega+\int_{\Omega} \alpha_{p}(1-f(I)) p p^{*} \mathrm{~d} \Omega=0 .
\end{aligned}
$$

$\forall \underline{v}^{*} \in\left(H_{0}^{1}(\Omega)\right)^{3}$ and $\forall p^{*} \in L^{2}(\Omega)$, with $f(I=1)=1$ and $f(I=0)=0$ and where the expression of the stress tensor is given by the constitutive Eq. (3). In the previous variational formulation, $H^{1}(\Omega)$ and $L^{2}(\Omega)$ denote the usual Sobolev and Lebesgue functional spaces and $H_{0}^{1}(\Omega)$ is the functional space of the velocities vanishing on the domain boundary $\Gamma$. The choice of the functions $f(I), \alpha_{v}$ and $\alpha_{p}$ is a key point to obtain a numerical scheme without numerical dissipation and with a low diffusion of the flow front (required to locate accurately the moving boundaries) [21]. 


\section{Updated Lagrangian meshless procedures: the natural neighbor Galerkin method}

\subsection{Natural neighbor interpolation}

The NEM has been applied to the simulation in solid mechanics by Sukumar and co-workers [22-24] and also by Doblaré and co-workers [25,26]. It can be considered as a member of the wide family of meshless methods for the solution of partial differential equations. Some examples of its application to the simulation of Newtonian fluids and non-Newtonian short fiber suspensions can be found in [27].

The NEM is based on the application of two classes of Natural Neighbor interpolation (Sibson [28,29] and Belikov et al. [30]) to the discretization of a variational formulation. In this paper, we will refer to the first one, although a deep study of the behavior of the second one can be found in [31]. Both methods share the main characteristics.

Sibson interpolation relies on the concepts of Delaunay triangulations and Dirichlet tesselations [32,33] of a set of nodes to build the shape functions (see Fig. 1). A Delaunay triangulation (tetrahedrization in three-dimensions) is the unique triangulation for a given set of nodes that satisfies the empty circumcircle criterion, that is, given the three nodes of a triangle (four nodes on a tetrahedron) and the circle (sphere) that passes through them, no one of the other nodes lies inside this circle (sphere). A Voronoi diagram or a Dirichlet tessellation is the dual structure of a Delaunay triangulation. For a given node $n_{I}$, the associated Voronoi cell is composed by all of the points which are closer to the node $n_{I}$ than to any other node. Formally,

$$
T_{I}=\left\{\underline{x} \in \mathbb{R}^{3}: d\left(\underline{x}, \underline{x}_{I}\right)<d\left(\underline{x}, \underline{x}_{J}\right), \quad \forall J \neq I\right\},
$$

where $T_{I}$ is the Voronoi cell and $d(\cdot, \cdot)$ represents the Euclidean distance. It is clear from Fig. 1 that the Delaunay triangulation is defined over the convex hull of the set of points.

In a similar way, the second-order Voronoi cell is defined as the locus of the points that have the node $n_{I}$ as the closest node and the node $n_{J}$ as the second closest node:

$$
T_{I J}=\left\{\underline{x} \in \mathbb{R}^{3}: d\left(\underline{x}, \underline{x}_{I}\right)<d\left(\underline{x}, \underline{x}_{J}\right)<d\left(\underline{x}, \underline{x}_{K}\right), \quad \forall K \neq I, J ; I \neq J\right\} .
$$

Thus, if a new point is added to a given cloud of points, the natural neighbor coordinates of this point $\underline{x}$ with respect to one of his neighbors $\underline{x}_{I}$ is the ratio of the cell $T_{I}$ that is transferred to $T_{x}$ when adding $\underline{x}$ to the initial cloud of points, to the total area of $T_{x}$. In other words, being $\kappa(\underline{x})$ and $\kappa_{I}(\underline{x})$ the Lebesgue measures of $T_{x}$ and $T_{x I}$ respectively, the natural neighbor coordinates of $\underline{x}$ with respect to the node $I$ is defined as

$$
\phi_{I}(\underline{x})=\frac{\kappa_{I}(\underline{x})}{\kappa(\underline{x})} .
$$
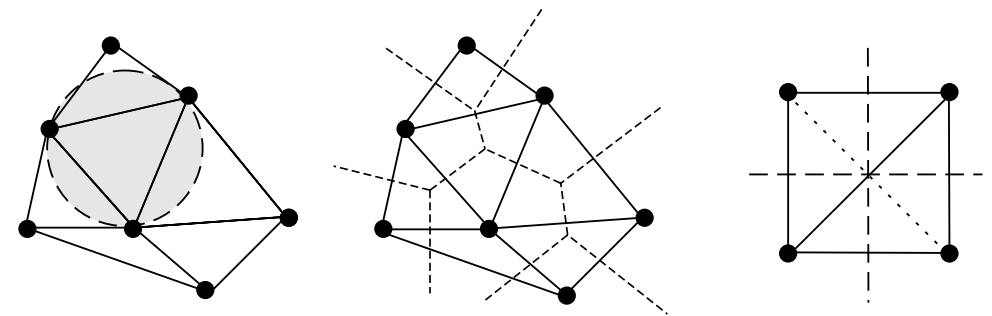

Fig. 1. Delaunay triangulation and Voronoi diagram of a cloud of points. On the right, an example of a degenerate case. 


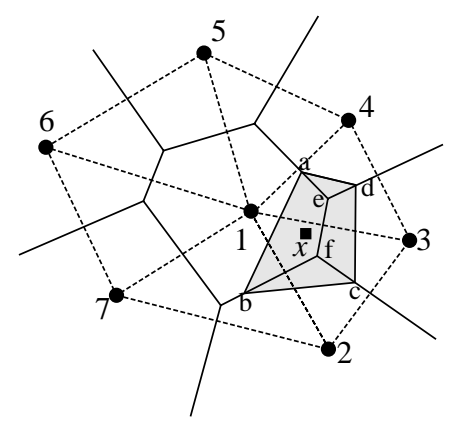

Fig. 2. Definition of the natural neighbor coordinates of a point $\boldsymbol{x}$.

In Fig. 2, this relationship may be written as

$$
\phi_{1}(\underline{x})=\frac{A_{\mathrm{abfe}}}{A_{\mathrm{abcd}}},
$$

being straightforward to prove that NE shape functions define a partition of unity. The resulting shape function is shown in Fig. 3.

A model variable $\boldsymbol{u}(\boldsymbol{u}$ represents a scalar, a vector or a tensor) can be approximated in 1D, 2D or 3D in the form:

$$
\boldsymbol{u}^{\mathrm{h}}(\underline{x})=\sum_{I=1}^{n} \phi_{I}(\underline{x}) \boldsymbol{u}_{I},
$$

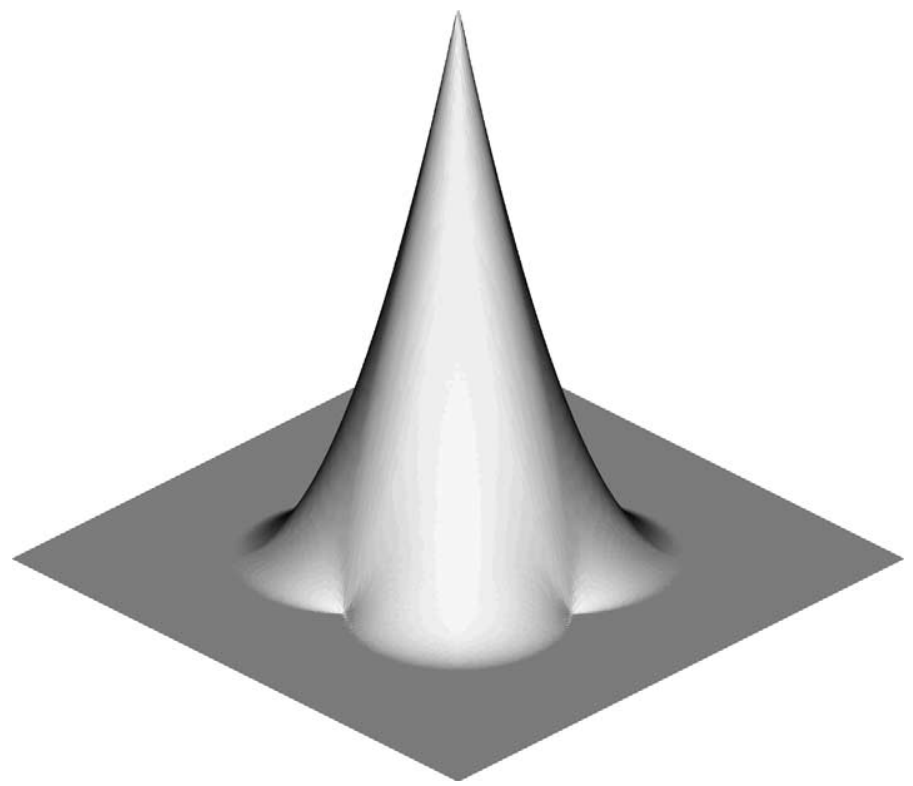

Fig. 3. Natural element shape function for a node surrounded of other six nodes [40]. 
where $\boldsymbol{u}_{I}$ represents the nodal values $\boldsymbol{u}_{I}=\boldsymbol{u}\left(\underline{x}_{I}\right)$ and $n$ the number of natural neighbors of each point $\underline{x}$. This leads to a $C^{0}$ interpolation, smooth everywhere except at the nodes [22], although it is also possible to build a $C^{1}$ interpolation [24]. Now, from the natural elements interpolation, any variational formulation can be discretized.

The NEM method has some interesting particularities, such as the Kronecker delta property of the shape functions

$$
\phi_{I}\left(\underline{x}_{J}\right)=\delta_{I J}
$$

Thus, the nodal parameters represent exactly the nodal values, which allows to impose prescribed values at certain nodes (essential boundary conditions for example) by direct substitution of the corresponding unknowns in the system of equations by those prescribed values.

The linear consistency of the interpolant can be demonstrated after the local coordinate expression

$$
\underline{x}=\sum_{I=1}^{n} \phi_{I}(\underline{x}) \underline{x}_{I},
$$

taking into account the partition of unity property. In other words, the natural neighbor interpolant can exactly reproduce a linear or a constant field. The shape functions thus calculated lead to standard linear finite elements shape functions if the considered point has three neighboring nodes, bilinear if the point has four neighbors and rational shape functions if the number of neighbors is five or more.

Another important property of the approximation described before is the ability to reproduce a linear interpolant along convex boundaries, as proved in Sukumar and co-workers [22,23]. This is not true in the general case of non-convex boundaries, where contributions of interior points are not negligible. Sukumar et al. [22] reported errors due to this fact of about $2 \%$ using non-uniform distributions of points, finer near the boundary.

In Cueto et al. [25,34], the issue of imposing essential boundary conditions is analyzed. Two main approaches to the problem are possible: the one in which the boundary of the domain is not explicitly defined (in a CAD sense) or the one in which it is. The first one leads to the $\alpha$-shape based natural element method $(\alpha$-NEM), while for the second a condition for the sampling density near the boundary has been proposed in order to ensure linear precision along it. In the next section, some ideas concerning the first approach are presented, since it is the one used in this work for the flow front tracking.

\subsection{The $\alpha$-shape based natural element method}

Recently, a modification of the way in which the natural neighbor interpolant is built has been proposed in order to achieve linear interpolation also over non-convex boundaries [25]. This modification is based on the concept of $\alpha$-shapes. These are a generalization of the concept of convex hull of a cloud of points and are widely used in the field of scientific visualization and computational geometry to "extract" the shape of a cloud of points. The concept was first developed by Edelsbrunner and co-workers [35,36] and can be resumed as follows.

In essence, an $\alpha$-shape is a polytope that is not necessarily convex nor connected, being triangulated by a subset of the Delaunay triangulation of the points. Thus, the empty circumcircle criterion holds. Let $N$ be a finite set of points in $\mathbb{R}^{3}$ and $\alpha$ a real number, with $0 \leq \alpha<\infty$. A $k$-simplex $\sigma_{T}$ with $0 \leq k \leq 3$ is defined as the convex hull of a subset $T \subseteq N$ of size $|T|=k+1$. Let $b$ be an $\alpha$-ball, that is, an open ball 
of radius $\alpha$. A $k$-simplex $\sigma_{T}$ is said to be $\alpha$-exposed if there exist an empty $\alpha$-ball $b$ with $T=\partial b \bigcap N$ where $\partial$ means the boundary of the ball. In other words, a $k$-simplex is said to be $\alpha$-exposed if an $\alpha$-ball that passes through its defining points contains no other point of the set $N$.

Thus, we can define the family of sets $F_{k, \alpha}$ as the sets of $\alpha$-exposed $k$-simplexes for the given set $N$. This allows us to define an $\alpha$-shape of the set $N$ as the polytope whose boundary consists on the triangles in $F_{2, \alpha}$, the edges in $F_{1, \alpha}$ and the vertices or nodes in $F_{0, \alpha}$.

A 3D simplicial complex is a collection, $\mathcal{C}$, of closed $k$-simplexes $(0 \leq k \leq 3)$ that satisfies:

(i) If $\sigma_{T} \in \mathcal{C}$ then $\sigma_{T^{\prime}} \in \mathcal{C}$ for every $T^{\prime} \subseteq T$.

(ii) The intersection of two simplexes in $\mathcal{C}$ is empty or is a face of both.

Each $k$-simplex $\sigma_{T}$ included in the Delaunay triangulation, $\mathcal{D}$, defines an open ball $b_{T}$ whose bounding spherical surface (in the general case) $\partial b_{T}$ passes through the $k+1$ points of the simplex. Let $\varrho_{T}$ be the radius of that bounding sphere, then, the family $G_{k, \alpha}$, is formed by all the $k$-simplexes $\sigma_{T} \in \mathcal{D}$ whose ball $b_{T}$ is empty and $\varrho_{T}<\alpha$. The family $G_{k, \alpha}$, does not necessarily form simplicial complexes, so Edelsbrunner and Mucke [36] defined the $\alpha$-complex, $\mathcal{C}_{\alpha}$, as the simplicial complex whose $k$-simplexes are either in $G_{k, \alpha}$, or else they bound $(k+1)$-simplexes of $C_{\alpha}$. If we define the underlying space of $\mathcal{C}_{\alpha}$, $\left|\mathcal{C}_{\alpha}\right|$, as the union of all simplexes in $\mathcal{C}_{\alpha}$, the following relationship between $\alpha$-shapes and $\alpha$-complexes is found:

$$
\mathcal{S}_{\alpha}=\left|\mathcal{C}_{\alpha}\right|, \quad \forall 0 \leq \alpha<\infty .
$$

If the natural neighborhood is limited to the case in which two nodes belong to the same triangle (tetrahedron) in a certain $\alpha$-complex, the linear interpolation property over convex boundaries is extended also to non-convex ones. This means that the Voronoi cells are no longer the basis for the computation of the shape function. Instead, we consider a cell

$$
T_{I}=\left\{\underline{x} \in \mathbb{R}^{3}: d\left(\underline{x}, n_{I}\right)<d\left(\underline{x}, n_{J}\right), \quad \forall J \neq I \wedge \sigma_{T} \in \mathcal{C}_{\alpha}(N)\right\} .
$$

$\mathcal{C}_{\alpha}(N)$ stands for an appropriate $\alpha$-complex, being $\sigma_{T}$ the $k$-simplex that form $n_{I}, n_{J}$ and any of the other point in the set $N$.

Consider as an example the regular gridded set of points $N$ and a non-convex boundary $\Gamma_{u}$ shown in Fig. 4. In order to guarantee that the solution at point $\underline{x}$ depends linearly on the solutions at nodes B and $\mathrm{C}$ we need to avoid the fact that A be a neighbor node of $\underline{x}$, even when $\underline{x}$ is approaching to node B. It is

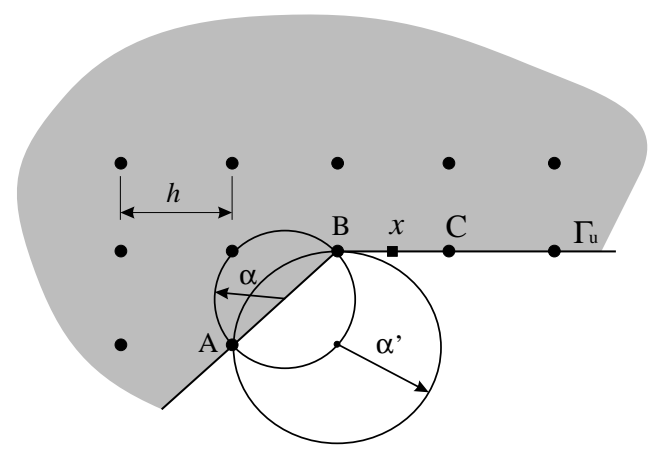

Fig. 4. Neighborhood in the context of $\alpha$-complexes. 


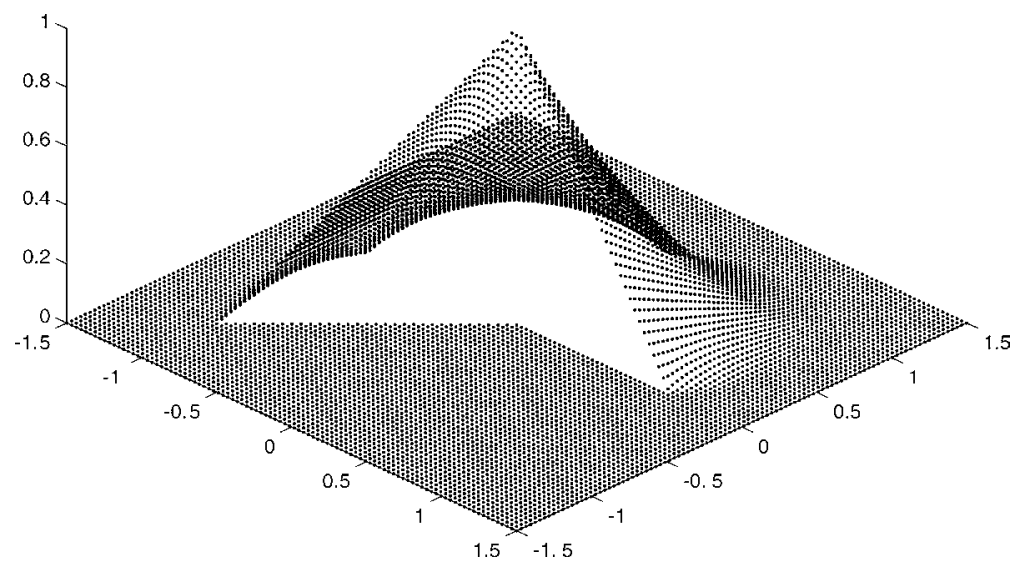

Fig. 5. Shape function associated to node B in Fig. 4.

easy to verify in Fig. 4 that if we take a value $\alpha<\alpha^{\prime}$, where $\alpha^{\prime}$ is the circumradius of the triangle defined by nodes A, B and the point $\underline{x}$ when $\underline{x}$ approaches to B, then the solution along the segment $\mathrm{B}-\mathrm{C}$ does not depend on the solution at node $\mathrm{A}$. Thus, the shape function related to the node $\mathrm{B}$ results as shown in Fig. 5, where the linearity of the shape function along the segments A-B and B-C can be noticed. A complete proof of this property can be found in [25,34].

Thus, the $\alpha$-NEM allows the flow front tracking without an explicitly description of the fluid domain boundary by using the $\alpha$-shape concept. The value of $\alpha$ can be adapted locally as a function of the nodal density in order to fit accurately the geometry details of the domain boundary [25].

\subsection{Mixed velocity-pressure interpolation in the resolution of the flow kinematics}

A mixed $C^{0}-C^{-1}$ natural element interpolation has been applied in incompressible elasticity as well as in the context of fluid mechanics problems. More details on the application of mixed NE interpolations in incompressible and nearly incompressible elasticity can be found in Cueto et al. [37].

In the formulation here presented, a $C^{0}$ interpolation scheme- smooth every where except at the nodeshas been chosen in the velocity field approximation, whereas a discontinuous $C^{-1}$ interpolation has been used in the pressure approximation:

$$
\begin{aligned}
& \underline{v}^{\mathrm{h}}(\underline{x})=\sum_{I=1}^{n} \phi_{I}(\underline{x}) \underline{v}_{I}, \\
& p^{\mathrm{h}}(\underline{x})=\sum_{I=1}^{n} \psi_{I}(\underline{x}) p_{I}=\sum_{I=1}^{n} \frac{1}{n} p_{I},
\end{aligned}
$$

where $\underline{v}_{I}$ and $p_{I}$ represent the nodal velocities and pressures, respectively, and $n$ is the number of natural neighbors of the considered point $\underline{x}$.

This kind of approximation does not verify the LBB condition, however, it has been shown that its behavior is very similar to that of the bilinear velocity-constant pressure finite element [37]. No spurious modes nor locking have been observed in all the simulations computed until now. 
Thus, with the fluid domain $\Omega_{\mathrm{f}}(t)$ extracted at time $t$ from the cloud of nodes by using the $\alpha$-shape technique, as described in the previous section, and the velocity and pressure natural element interpolation defined by Eqs. (29) and (30), we can proceed to a standard discretization of the mixed variational formulation of the flow equations

$$
\begin{aligned}
& \int_{\Omega_{\mathrm{f}}(t)} \underline{\sigma}: \underline{D}^{*} \mathrm{~d} \Omega=0, \\
& \int_{\Omega_{\mathrm{f}}(t)} \operatorname{Div} \underline{v} p^{*} \mathrm{~d} \Omega=0,
\end{aligned}
$$

with

$$
\underline{\underline{\sigma}}=-p \underline{\underline{I}}+2 \mu\left\{\underline{\underline{D}}+N_{p} \operatorname{Tr}(\underline{\underline{a}} \underline{\underline{D}}) \underline{\underline{a}}\right\},
$$

where a null traction is assumed on the flow front and a prescribed velocity is enforced on the other part of the fluid domain boundary. Finally, in the kinematics resolution stage, the fiber orientation described from the second-order orientation tensor $\underline{\underline{a}}$ is assumed known at the nodes at present time $\underline{a}_{I}^{t}$. The value of $\underline{\underline{a}}$ at the integration points used to evaluate Eqs. (31) and (32) is computed by using the natural element interpolation

$$
\underline{\underline{a}}^{t}(\underline{x})=\sum_{I=1}^{n} \phi_{I}(\underline{x}) \underline{\underline{a}}_{I}^{t} .
$$

\subsection{Integration of advection equations}

With the flow kinematics $\underline{v}^{t}(\underline{x}) \equiv \underline{v}(\underline{x}, t)$ computed at time $t$, from Eqs. (31)-(33), both the position of the nodes and the fiber orientation can be updated simultaneously using the method of characteristics. The simplest explicit and first-order updating evaluates

$$
\underline{x}_{I}^{t+\Delta t}=\underline{x}_{I}^{t}+\underline{v}_{I}^{t} \Delta t, \quad \forall I
$$

and from Eq. (8)

$$
\underline{\underline{a}}_{I}^{t+\Delta t}=\underline{\underline{a}}_{I}^{t}+\left\{\underline{\Omega}_{I}^{t} \underline{\underline{a}}_{I}^{t}-\underline{\underline{a}}_{I}^{t} \stackrel{\Omega}{=}_{I}^{t}+k \underline{\underline{D}}_{I}^{t} \underline{\underline{a}}_{I}^{t}+k \underline{\underline{a}}_{I}^{t} \underline{D}_{I}^{t}-2 k \operatorname{Tr}\left(\underline{\underline{a}}_{I}^{t} \underline{\underline{D}}_{I}^{t}\right) \underline{\underline{a}}_{I}^{t}-D_{r}\left(\underline{\underline{a}}_{I}^{t}-\frac{1}{2} \underline{I}\right)\right\} \Delta t, \quad \forall I
$$

where $\underline{D}_{I}^{t}$ and $\Omega_{I}^{t}$ are the symmetric and skew-symmetric components of the velocity gradient tensor, respectively, both computed at time $t$ in the node $\underline{x}_{I}$. Even if higher-order explicit or implicit techniques are available, due to the small time steps used in the updated Lagrangian strategy, no significant difference has been noticed by using higher order schemes (e.g. fourth-order Runge-Kutta) for integrating the orientation equation.

There is only one difficulty in the application of the orientation updating given by Eq. (36), which is related to the non-derivability of the natural element shape functions at their definition nodes. Thus, we can evaluate the velocity gradient tensor from the expression

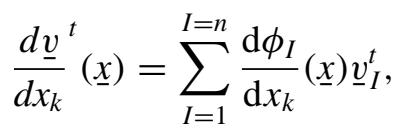

everywhere except at the nodes. 
In order to circumvent this problem, two simple possibilities exist. In the first one, used in this work, Eq. (36) is applied to update the fiber orientation at the quadrature points, which are in fact used to integrate the variational formulation of the equations of motion. The orientation at the new nodal positions (resulting from the integration of Eq. (35)) is obtained using the least square procedure proposed by Hinton and Campbell [38], to minimize the relative difference between the orientations obtained from the nodes and the original ones stored at quadrature points. The main drawback of this technique is that a certain amount of numerical diffusion is introduced in the projection between quadrature and nodal points.

Other possibility for avoiding the problems related to the non derivability of the natural element shape functions at the nodal positions, lies in defining the average of the velocity gradient in the Voronoi cell $T_{I}$

$$
\underline{\widetilde{v}}, k_{T_{I}}\left|T_{I}\right|=\int_{T_{I}} \underline{v}_{, k} \mathrm{~d} \Omega=\int_{\partial T_{I}} \underline{v} n_{k} \mathrm{~d} S,
$$

where $n_{k}$ is the $k$-component of $\underline{n}, T_{I}$ is the Voronoi cell associated with the node $\underline{x}_{I}$ whose volume is denoted by $\left|T_{I}\right|$ and its boundary by $\partial T_{I}$. This kind of averaging was introduced in the framework of the natural element approximation in [39]. Thus, the fiber orientation updating results in

$$
\underline{\underline{a}}_{I}^{t+\Delta t}=\underline{\underline{a}}_{I}^{t}+\left\{\underline{\tilde{\Omega}}_{I}^{t} \underline{\underline{a}}_{I}^{t}-\underline{\underline{a}}_{I}^{t} \underline{\tilde{\Omega}}_{I}^{t}+k \underline{\underline{D}}_{I}^{t} \underline{\underline{a}}_{I}^{t}+k \underline{\underline{a}}_{I}^{t} \underline{\tilde{D}}_{I}^{t}-2 k \operatorname{Tr}\left(\underline{\underline{a}}_{I}^{t} \tilde{\tilde{D}}_{I}^{t}\right) \underline{\underline{\underline{S}}}_{I}^{t}-D_{r}\left(\underline{\underline{a}}_{I}^{t}-\frac{1}{2} \underline{\underline{I}}\right)\right\} \Delta t, \quad \forall I
$$

where

$$
\underline{\underline{D}}=\frac{1}{2} \widetilde{\operatorname{Grad} \underline{v}}+(\widetilde{\operatorname{Grad} \underline{v}})^{\mathrm{T}}
$$

and

$$
\tilde{\tilde{\Omega}}=\frac{1}{2} \widetilde{\operatorname{Grad} \underline{v}}-(\widetilde{\operatorname{Grad} \underline{v}})^{\mathrm{T}} .
$$

The comparison between the results obtained using both procedures is a work in progress.

\section{Numerical examples}

\subsection{Filling a cavity}

Fig. 6 depicts the cavity whose filling process will be simulated using the natural element meshless method just described (in [21], computational aspects related to the fixed mesh simulation described in Section 2 were explained). The suspension viscosity is fixed to $10^{3} \mathrm{~Pa}$ s, the fibers are assumed to have a quasi-infinite aspect ratio, $k=1$, being the particle number of 1 or $10, N_{p}=1$ or $N_{p}=10$, and all

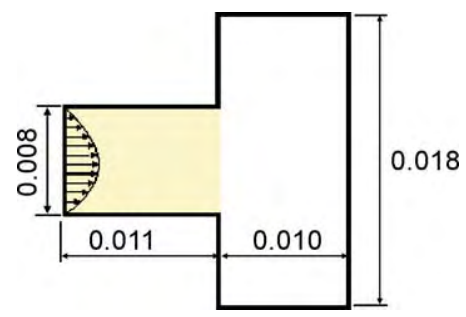

Fig. 6. Cavity geometry. 

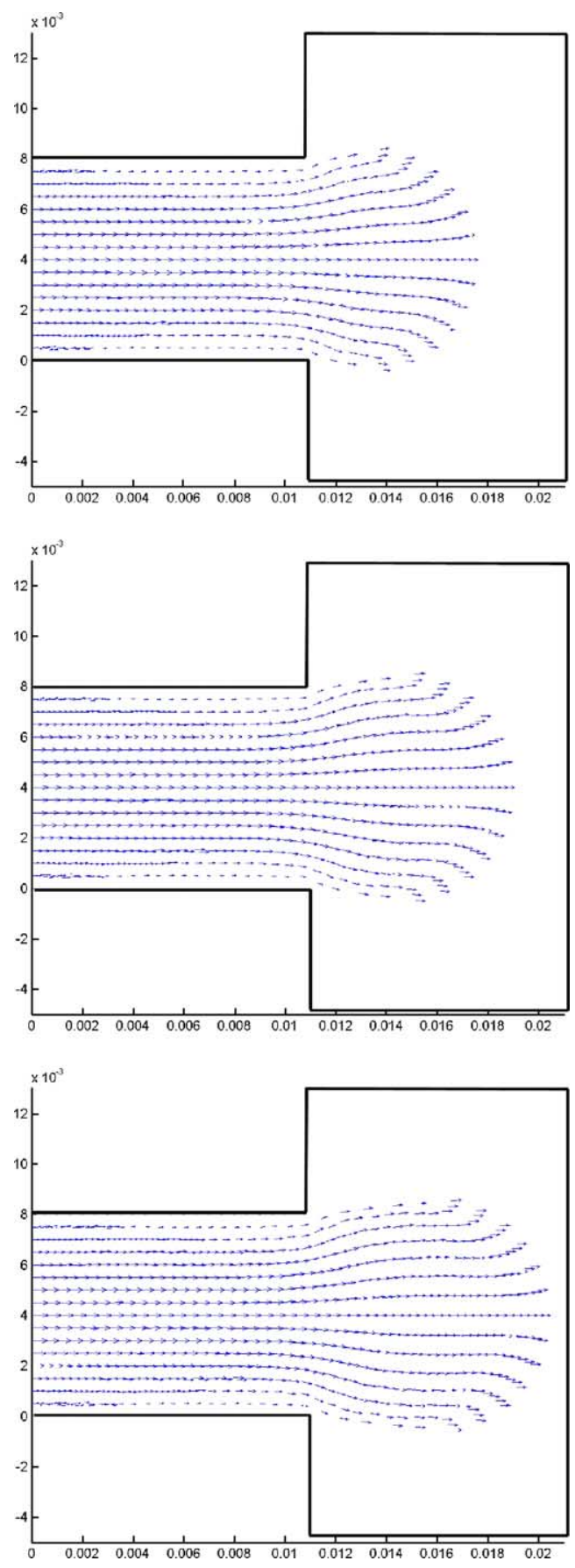

Fig. 7. $\alpha$-NEM filling process simulation: flow kinematics during the extrusion stage $\left(N_{p}=1\right)$. 
diffusion effects are neglected $\left(D_{r}=0\right)$. At time $t=0$, the suspension is assumed filling the inlet cavity (as shown in Fig. 6) with an initial isotropic fiber orientation distribution $(\underline{\underline{a}}(t=0)=\underline{\underline{I}} / 2)$.

Figs. 7-9 show a filling process sequence obtained using the $\alpha$-NEM. The main particularity of this numerical example is that when the filling process starts, the flow is not affected by the mold walls, as noticed in Fig. 7, and in consequence it consists actually in an extruded flow. The updated Lagrangian meshless technique allows then an accurate description of the flow front as well as an accurate integration of the fiber orientation equation using the method of characteristics. Fig. 10 shows the highly distorted Delaunay triangulation at an intermediate filling time, which proves clearly the no significant dependence of the natural element interpolation accuracy on the nodal distribution. This fact constitutes the essential difference between the finite element method and the NEM, proving that the natural element strategy seems to be a very interesting alternative to treat 3D models in large transformations, where the frequent remeshing required by the finite element method, in the context of Lagrangian descriptions, still remains a difficult matter.

In order to check the ability of the $\alpha$-shape technique to extract accurately the fluid volume at each time, we compare in Fig. 11 the volume of the fluid domain automatically extracted from the cloud of nodes using the $\alpha$-shape concepts with the volume of fluid really injected. Fig. 12 represents the difference between both volumes with respect to the injected one. We can notice the excellent accuracy (the error is lower than $1 \%$ during the main part of the filling process, and higher values only appear in the last time steps when the domain is nearly fully filled) in spite of the reduced number of nodes used in the simulation. Obviously, this error decreases as the nodal density increases, as a direct consequence of the improvement in the flow front representation and the convergence of the natural element discretization technique.

The numerical strategy used decouples the flow kinematics resolution problem from the fiber orientation evolution problem. The convergence of the first one, which defines an elliptic anisotropic Stokes problem, was proved in $[22,23,25,40]$. With respect to the second one, the stability of its integration using the method of characteristics only requires a small enough time step. The convergence and stability analysis of the coupled problem is a more difficult matter which requires a deeper work, but in any case the mass conservation noticed and the qualitative agreement with the fixed mesh Eulerian simulations seem to indicate a good behavior.

Finally, Fig. 13 depicts the flow kinematics obtained using a fiber suspension characterized by a higher particle number $N_{p}=10$. As expected, for the first iterations of the filling process, when the flow is actually extruded, the die swelling increases with the particle number (the normal stress differences increase with the parameter $N_{p}$ ).

Concerning the computing time, the NEM shape function computation is about two times slower than traditional finite elements. However, it must be noted that this comparison takes into account the neighbor search, which is in some form equivalent to the user time employed in mesh generation [22].

\subsection{Interacting flows}

In this example, we analyze the induced orientation when two flow fronts, with and without parallel fiber orientation, meet. In the simulation, we have considered $N_{p}=1$ and in order to evidence the non-mixing during the welding the nodes arriving in each flow have been colored differently. Sliding boundary conditions are imposed in the inflow channel walls and a sticking contact in the outflow channel walls. 

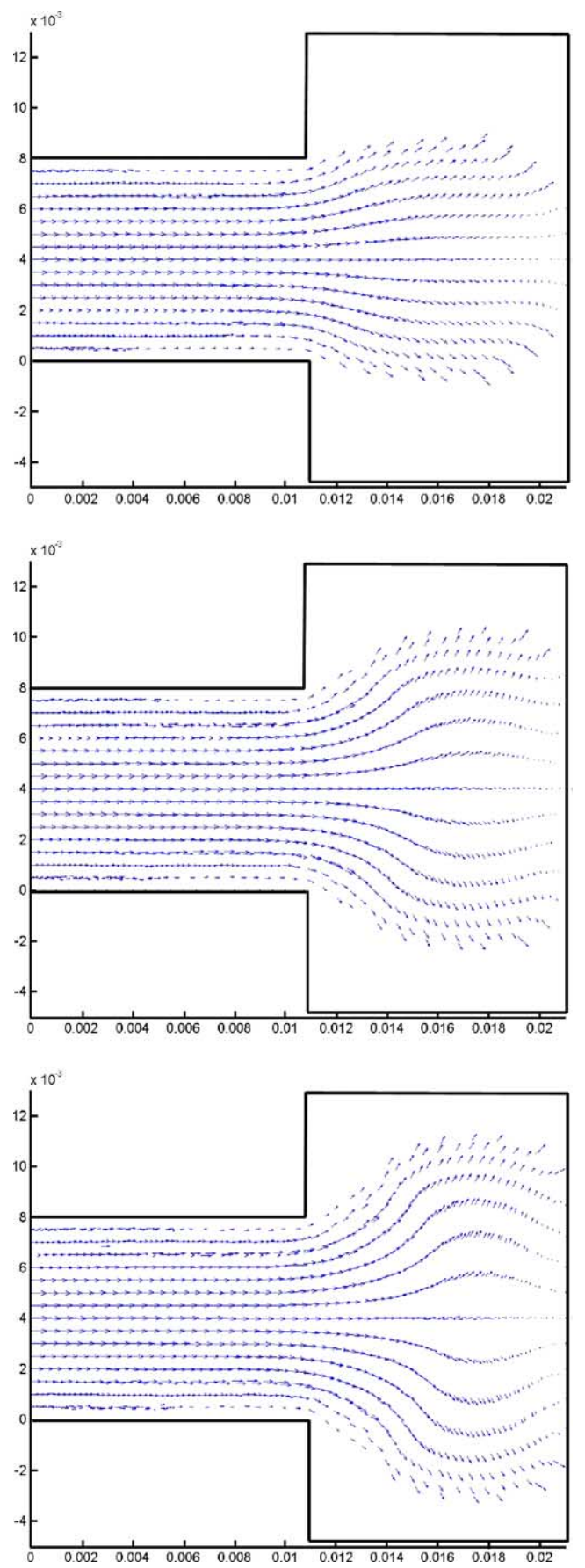

Fig. 8. $\alpha$-NEM filling process simulation: flow kinematics during the mold filling $\left(N_{p}=1\right)$. 

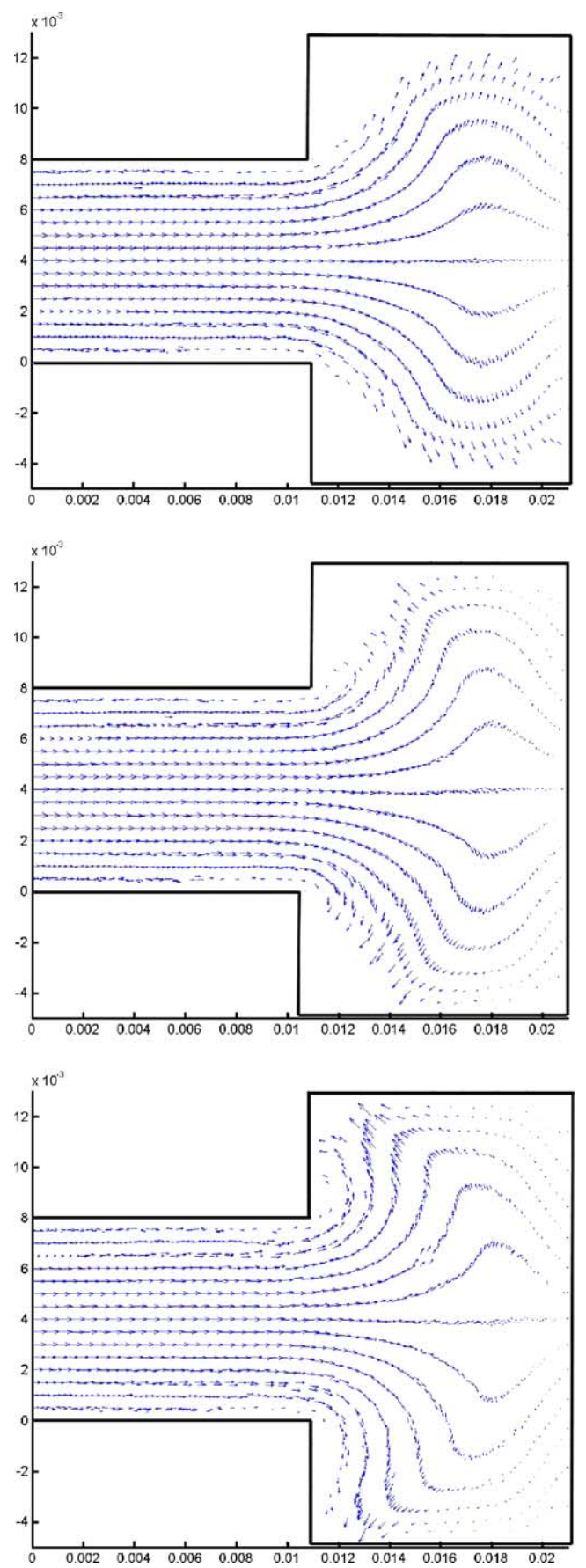

Fig. 9. $\alpha$-NEM filling process simulation: last time steps $\left(N_{p}=1\right)$. 


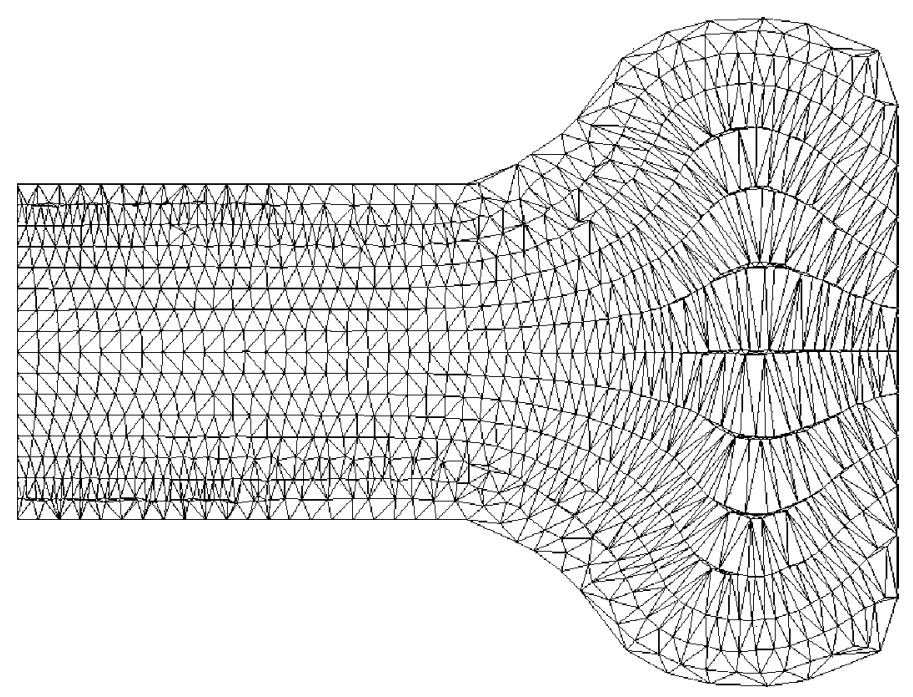

Fig. 10. Delaunay triangulation in an intermediate time of the filling process.

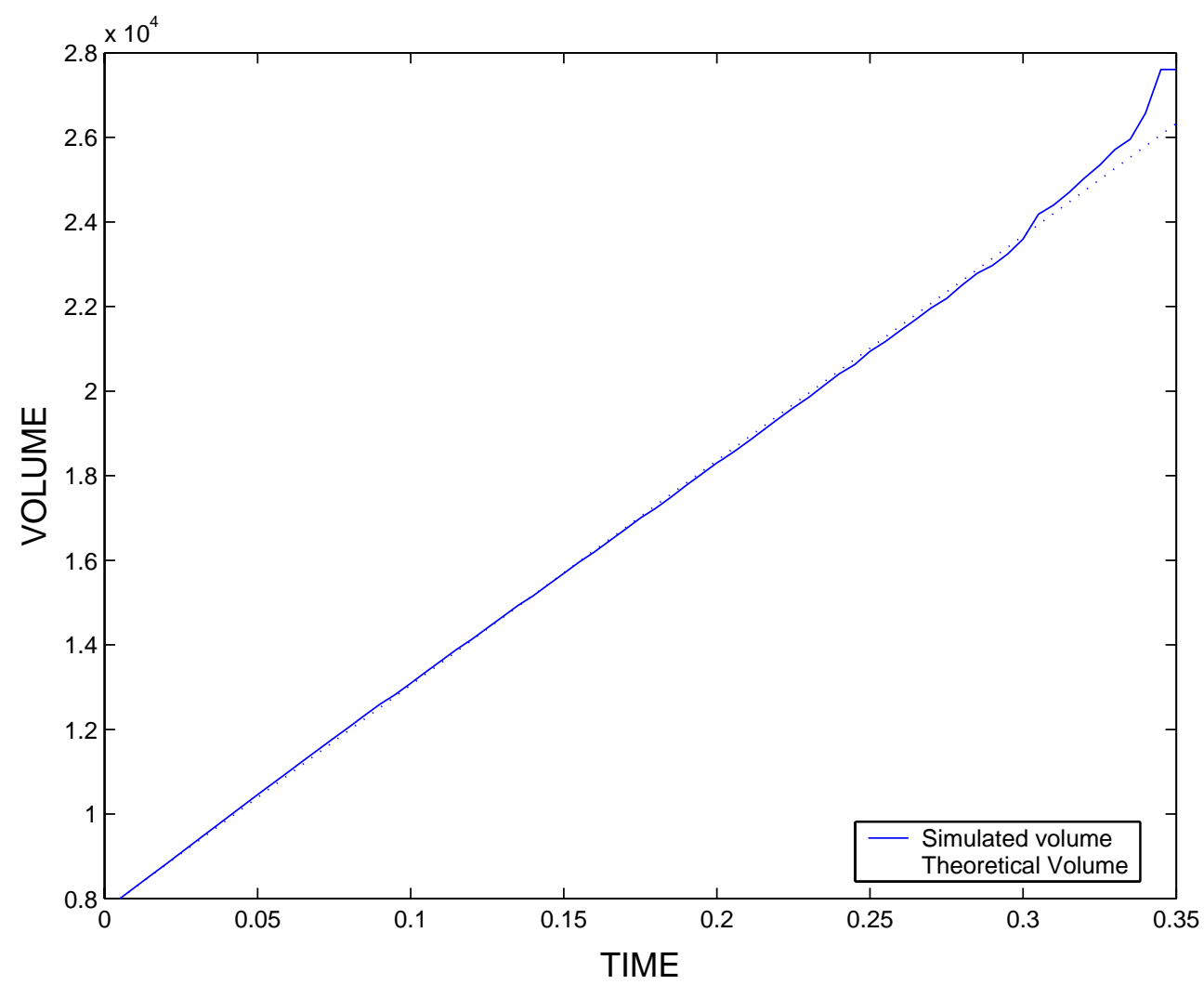

Fig. 11. $\alpha$-NEM filling process simulation: evolution of the fluid domain volume. 


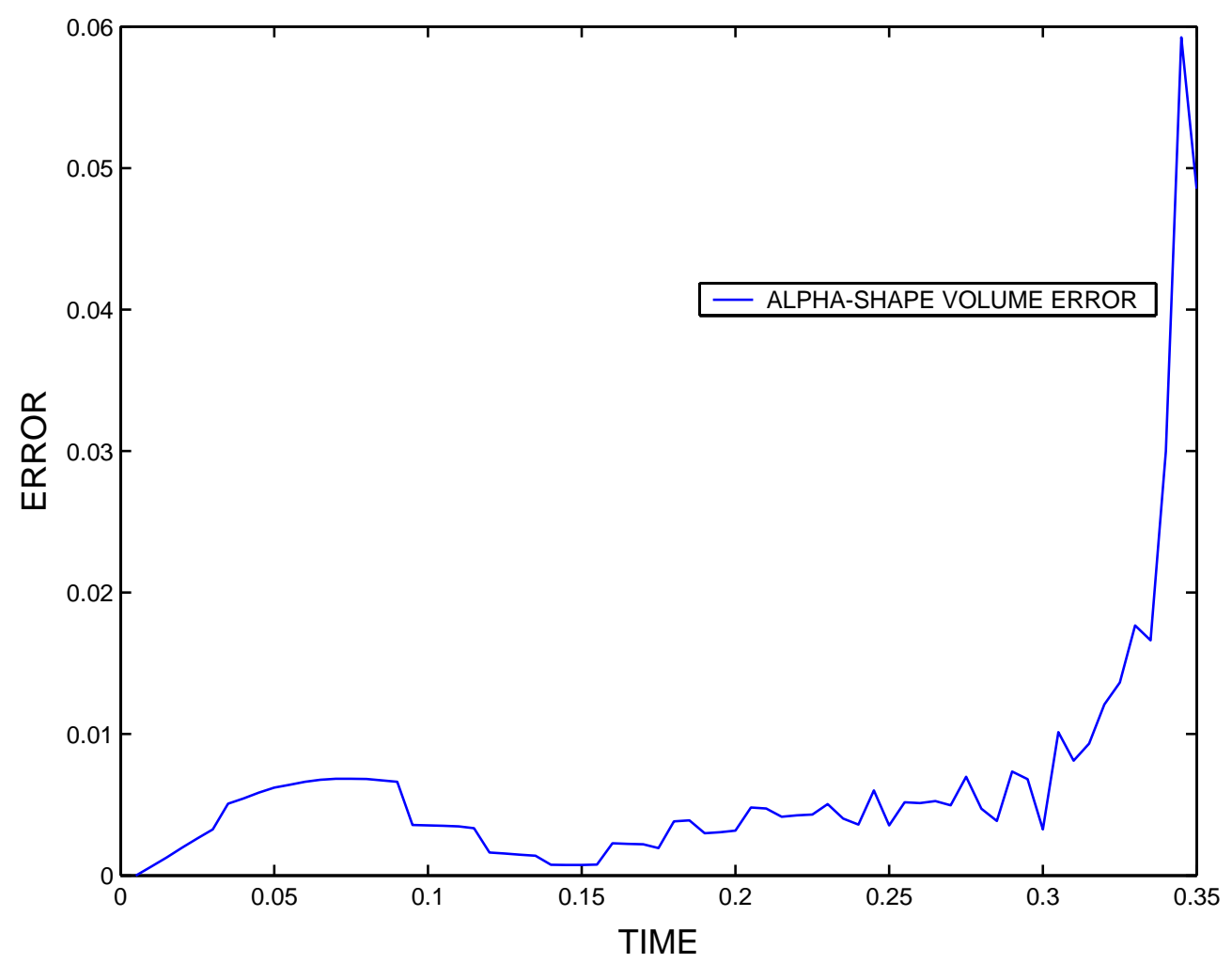

Fig. 12. $\alpha$-NEM filling process simulation: difference between the computed and the injected volumes with respect to the injected one.

In the first case, as noticed in the flow sequence depicted in Figs. 14 and 15, the flow kinematics and the fiber orientation are perfectly symmetric. Moreover, as discussed in a former paper [40], the flow fronts welding is assumed taking place when they approach a distance lower than the $\alpha$ parameter, which is itself of $h$ order ( $h$ being a characteristic distance between nodes). This result proves that the error introduced in the fronts welding is in the order of $h$, and then the convergence of the proposed strategy.

The flow fronts welding in the case of non parallel fiber orientation is depicted in Figs. 16 and 17. In this case, we can notice that when the fronts welding occurs, an isotropic orientation state results on the welding line, which is in agreement with the expected distribution resulting from an average at the quadrature points between the fiber orientations of the incident flows.

Finally, Figs. 18 and 19 show the interaction between two flows in a non-confined domain. In all the cases, we can notice that due to the fact of computing the fiber orientation evolution at the integration points, with the associated projections between nodes and quadrature points, some amount of numerical diffusion is introduced in the solution on the welding line as well as in the neighborhood of the zones where the fiber orientation evolves very fast, e.g. the flow corners. We expect to limit these undesired effects by changing the nodal gradient of velocity (that cannot be computed in the context of the NEM) by the average of the gradient of velocity defined by Eq. (38) which will that after be assigned to the associated node for integrating Eq. (36). The analysis of this approach, whose first results seem to be very promising, is in progress. 

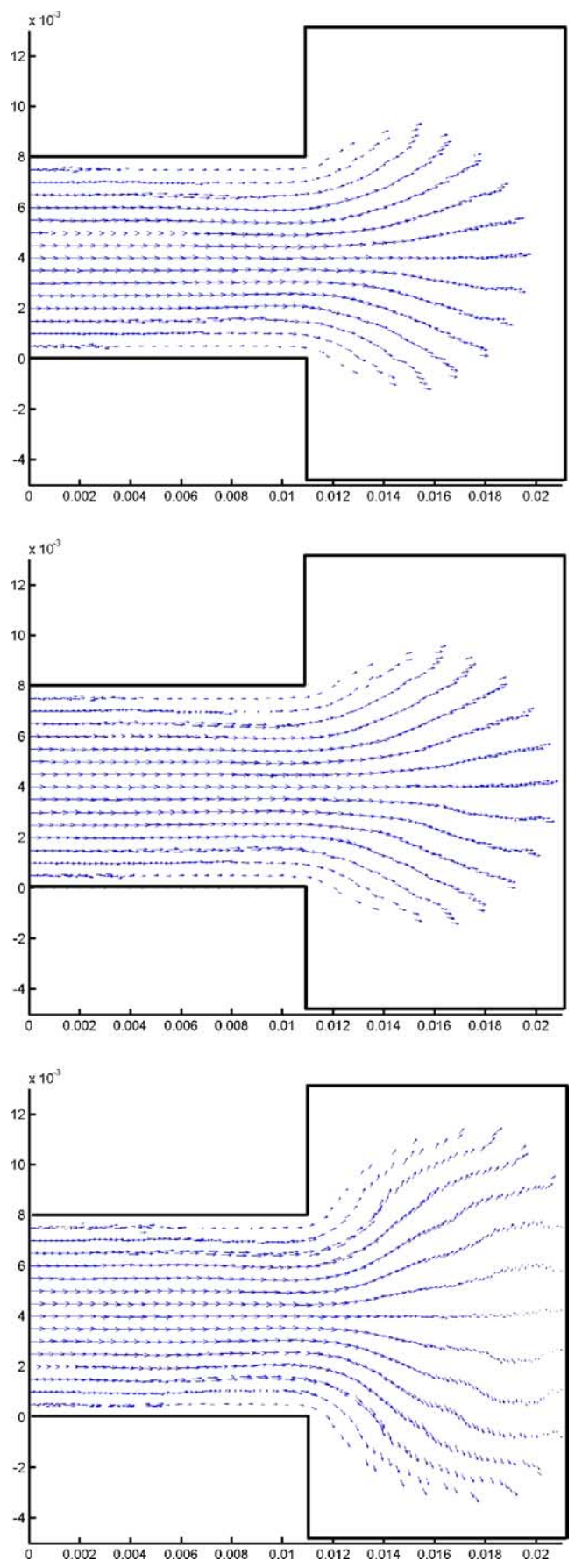

Fig. 13. $\alpha$-NEM filling process simulation: flow kinematics for a higher particle number. 

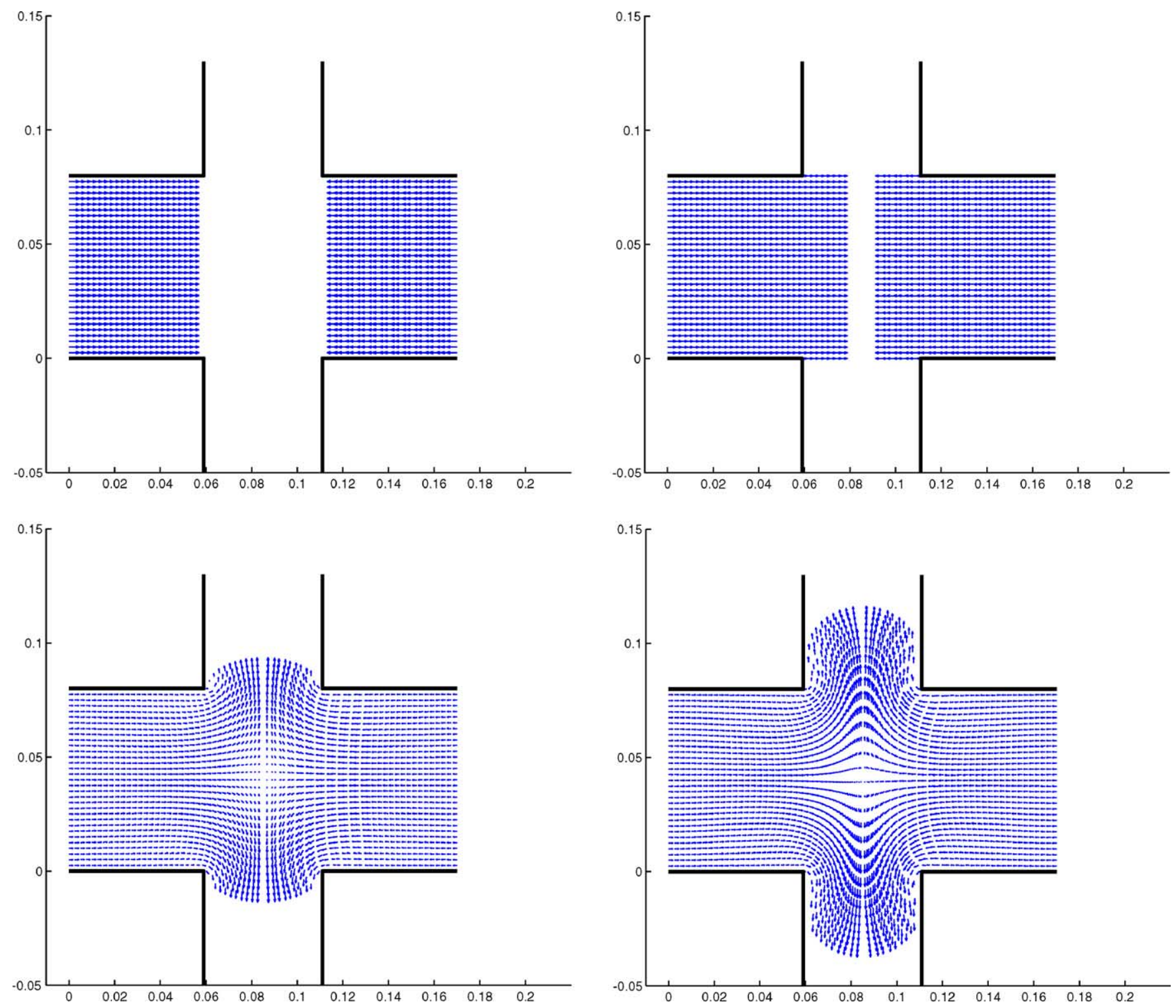

Fig. 14. Flow fronts welding: flow kinematics for parallel fiber orientation. 

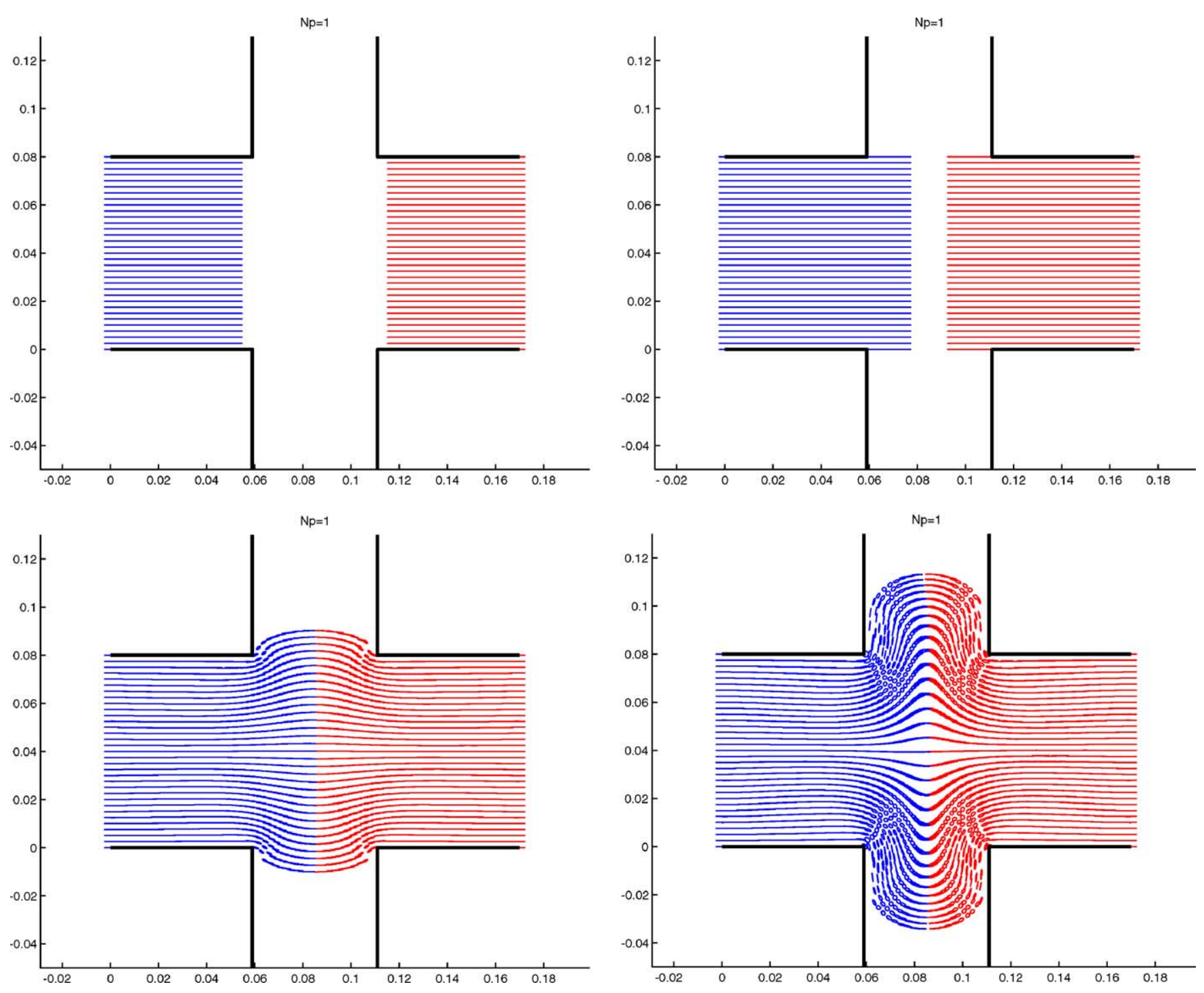

Fig. 15. Flow fronts welding: fiber orientation for parallel fiber orientation. 

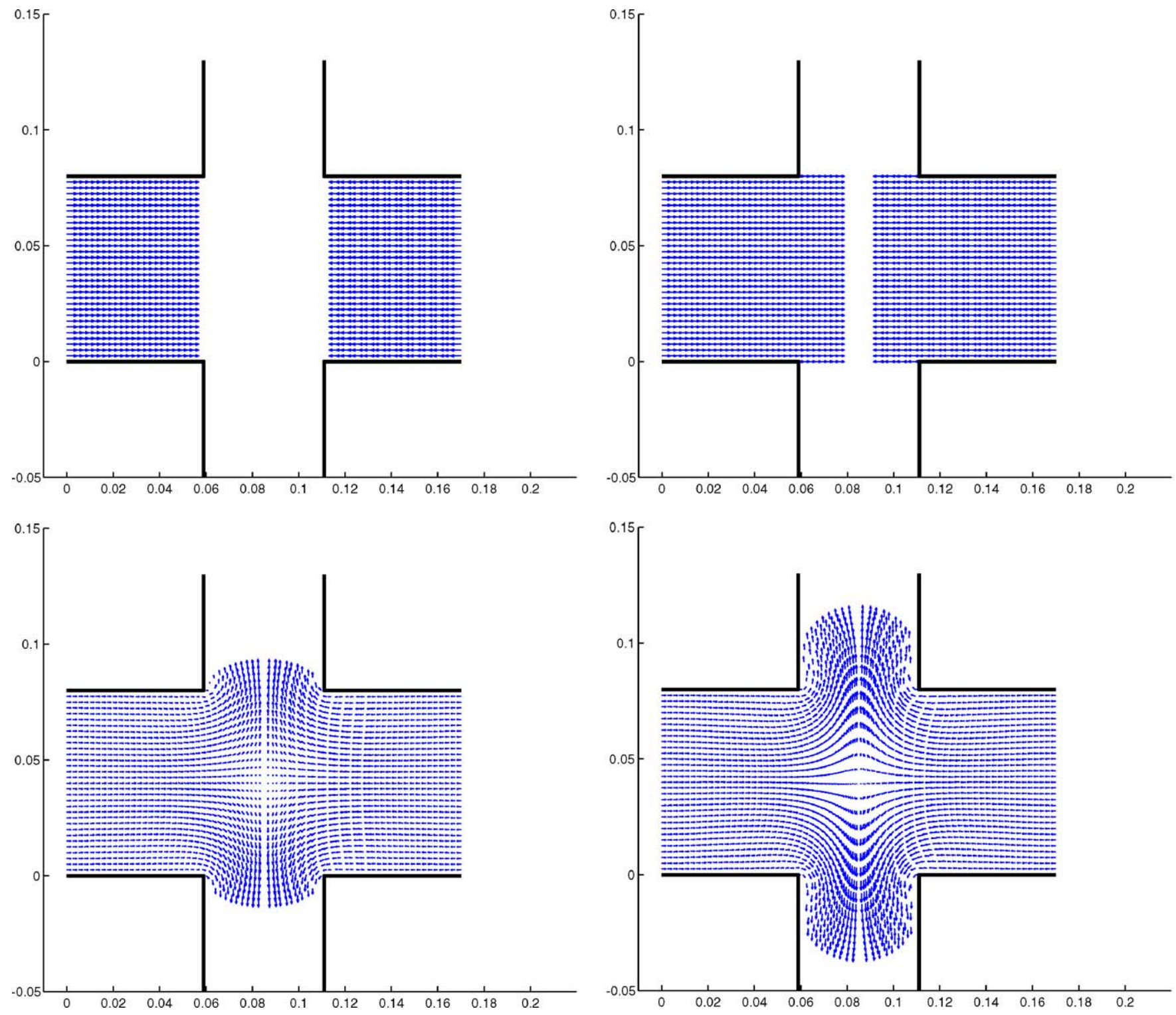

Fig. 16. Flow fronts welding: flow kinematics for non-parallel fiber orientation. 

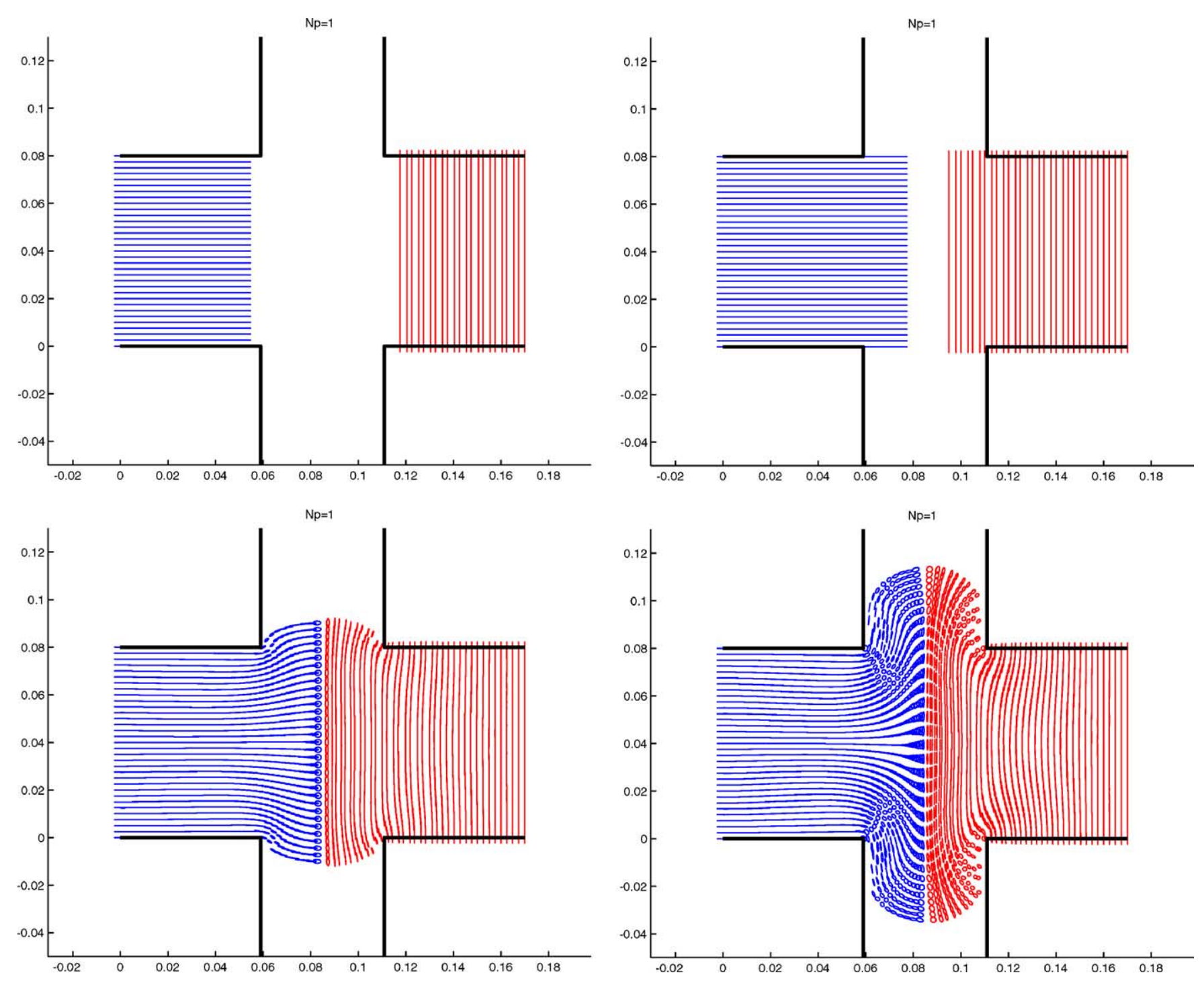

Fig. 17. Flow fronts welding: fiber orientation for non-parallel fiber orientation. 

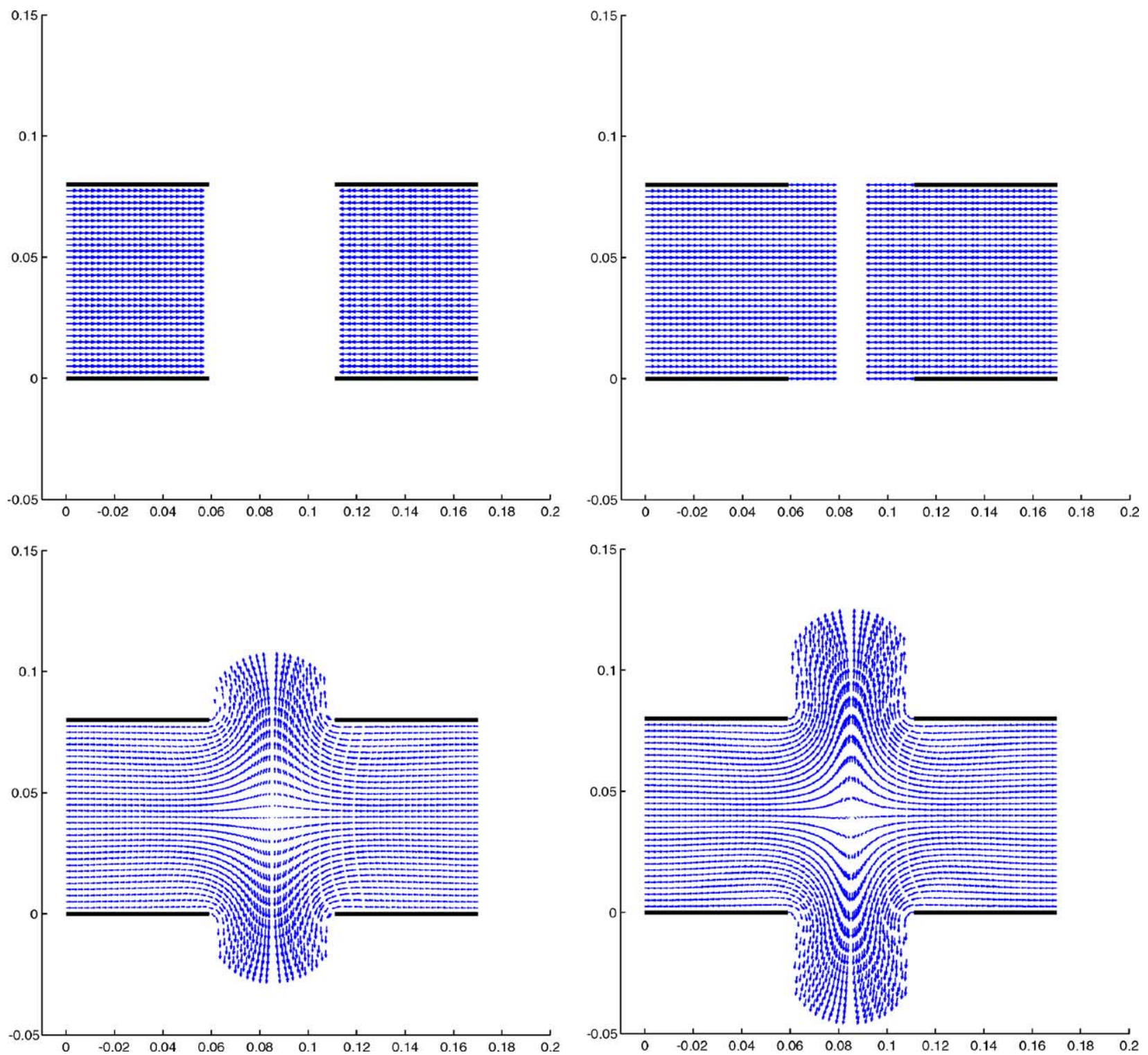

Fig. 18. Flow fronts welding: flow kinematics in an unbounded domain. 

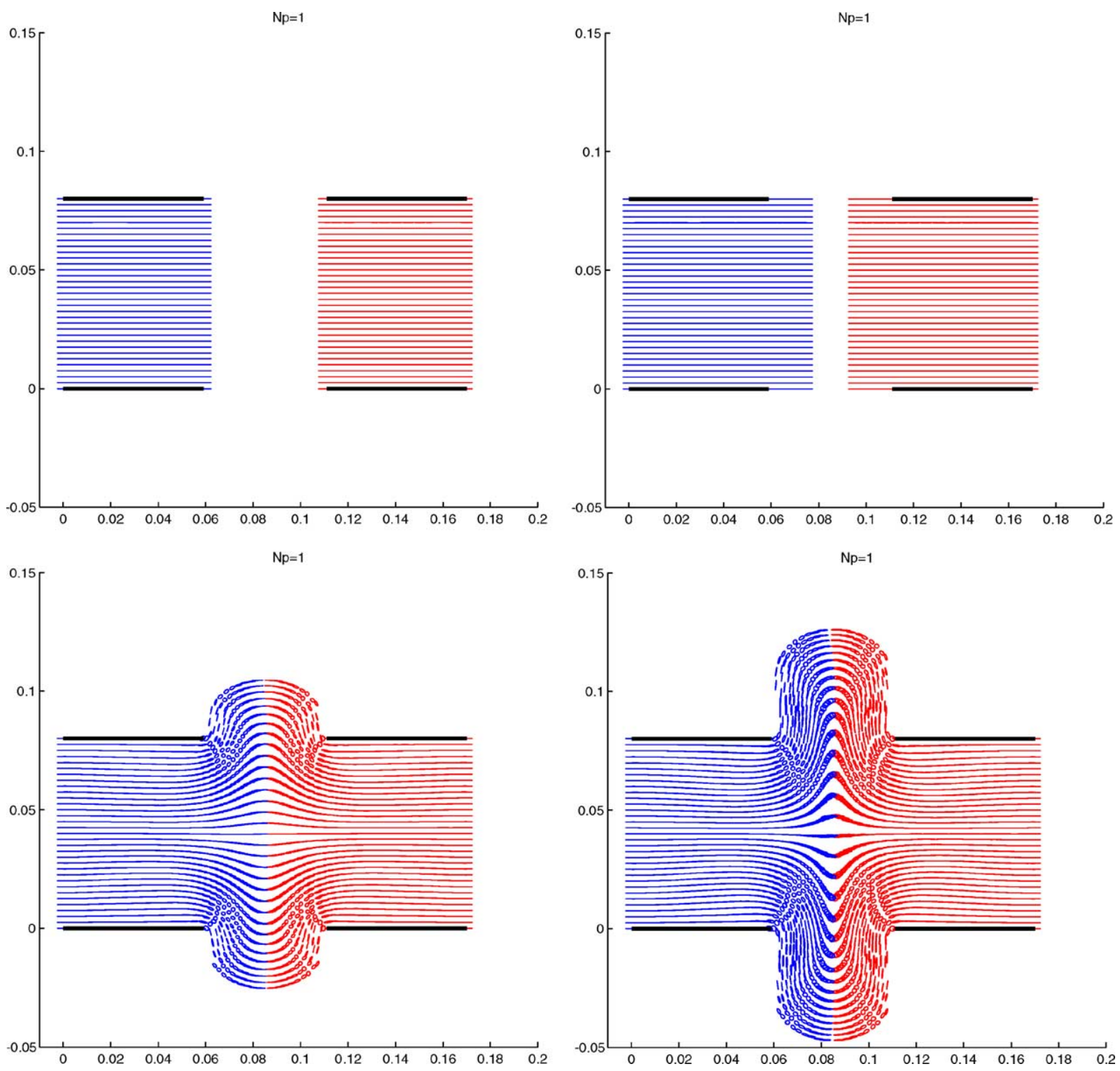

Fig. 19. Flow fronts welding: fiber orientation in an unbounded domain.

\section{Conclusions}

In this paper, we have refereed to two different strategies for treating problems involving moving or free boundaries. The first one uses a VOF fixed mesh description. This type of modeling avoids the necessity of remeshing, however, the treatment of advection problems related to the fluid history (evolution of the anisotropic viscosity due to the fiber orientation evolution) becomes a delicate matter. Thus, adapted discretization techniques for hyperbolic equations are required in order to solve accurately 
the advection equations governing the orientation evolution of fibers immersed in the flow. It is well known that Lagrangian description is suitable to take into account accurately the fluid history (integrating transport equations along the flow trajectories, using for example the method of characteristics). However, in this case, large mesh distortions occur, which requires frequent remeshing. In the $2 \mathrm{D}$ case, efficient remeshing strategies exist, which is not the general case in 3D.

The NEM is an attractive alternative, because its ability to integrate transport equations and its meshless character. The approximation of the solution is made from a nodal description, without geometrical restrictions about the relative position of the nodes. In this form, the introduction or elimination of nodes is a trivial task.

In this paper, we have presented the capabilities of the NEM to simulate some flows more or less complex. However, in our opinion, it is in the context of 3D applications where the NEM will be a real alternative to the more classical techniques based in the use of fixed mesh. The extension to other non-Newtonian behaviors and the treatment of 3D models, are some of the works in progress.

\section{References}

[1] C. Givler, M.J. Crochet, R.B. Pipes, Numerically Predicted Fiber Orientation in Dilute Suspensions, NUMIFORM, Pineridge Press, 1982, pp. 559-575.

[2] J. Rosenberg, M. Den, R. Keunings, Simulation of non-recirculating flows of dilute fiber suspensions, J. Non-Newtonian Fluid Mech. 37 (1990) 317-345.

[3] G. Ausias, Etude de l'Extrusion de Tubes en Polymères Thermoplastiques Chargés de Fibers Courtes, Ph.D. thesis, ENSMP, 1991.

[4] M.C. Altan, S.I. Guçeri, R.B. Pipes, Anisotropic channel flow of fiber suspensions, J. Non-Newtonian Fluid Mech. 42 (1992) 65-83.

[5] K. Chiba, K. Nakamura, Numerical solution of fiber suspensions flow through a complex channel, J. Non-Newtonian Fluid Mech. 78 (1998) 167-185.

[6] A. Poitou, F. Chinesta, R. Torres, Numerical simulation of the steady recirculating flows of fibers suspensions, J. Non-Newtonian Fluid Mech. 90 (2000) 65-80.

[7] F. Dupret, V. Verleye, Modeling the flow of fiber suspensions in narrow gaps, in: D.A. Siginer, D. De Kee, R.P. Chabra (Eds.), Advances in the Flow and Rheology of Non-Newtonian Fluids, Elsevier, Amsterdam, 1999, pp. 1347-1398.

[8] T. Belytschko, Y. Krongauz, D. Organ, M. Fleming, P. Krysl, Meshless methods: an overview and recent developments, Comput. Methods Appl. Mech. Eng. 139 (1996) 3-47.

[9] E. Cueto, El Método de los Elementos Naturales Basado en Formas $\alpha$. Ph.D. dissertation, Universidad de Zaragoza, 2001.

[10] G.L. Hand, A theory of anisotropic fluids, J. Fluid Mech. 13 (1962) 33-46.

[11] G.K. Batchelor, Slender-body theory for particles of arbitrary cross-section in Stokes flow, J. Fluid Mech. 44 (1970) 419-440.

[12] E.J. Hinch, L.G. Leal, Constitutive equations in suspension mechanics, part I, J. Fluid Mech. 71 (1975) $481-495$.

[13] E.J. Hinch, L.G. Leal, Constitutive equations in suspension mechanics, part II, J. Fluid Mech. 76 (1976) $187-208$.

[14] F. Meslin, Propriétés Rhéologiques des Composites Fibers Courtes á l'Etat Fondu, Ph.D. thesis, Ecole Normale Superieure de Cachan, 1997.

[15] Ch.L. Tucker Jr., Flow regimes for fiber suspensions in narrow gap, J. Non-Newtonian Fluid Mech. 39 (1991) $239-268$.

[16] S.G. Advani, Ch.L. Tucker Jr., Closure approximations for three-dimensional structure tensors, J. Rheol. 34 (1990) $367-386$.

[17] V. Verleye, F. Dupret, Prediction of fibre orientation in complex injection moulded parts, in: Developments in Non-Newtonian Flows, AMD vol. 175, ASME, New York, 1993, pp. 139-163.

[18] F. Chinesta, G. Chaidron, A. Poitou, On the solution of Fokker-Planck equations in steady recirculating flows involving short fiber suspensions, J. Non-Newtonian Fluid Mech. 113 (2003) 97-125.

[19] O. Pironneau, Finite Element Method for Fluids, Wiley, New York, 1989.

[20] E. Pichelin, T. Coupez, Finite element solution of the 3D mold filling for viscous incompressible fluid, Comput. Methods Appl. Mech. Eng. 163 (1998) 359-371. 
[21] J. Azaiez, K. Chiba, F. Chinesta, A. Poitou, State-of-the-art on numerical simulation of fiber-reinforced thermoplastics forming processes, Arch. Comput. Methods Eng. 9 (2) (2002) 141-198.

[22] N. Sukumar, B. Moran, T. Belytschko, The natural element method in solid mechanics, Int. J. Numer. Methods Eng. 43 (5) (1998) 839-887.

[23] N. Sukumar, The natural element method in solid mechanics, Ph.D. thesis, Northwestern University, Evanston, IL, 1998.

[24] N. Sukumar, B. Moran, $C^{1}$ Natural neighbor interpolant for partial differential equations, Numer. Methods Part. Differ. Equat. 15 (4) (1999) 417-447.

[25] E. Cueto, M. Doblaré, L. Gracia, Imposing essential boundary conditions in the natural element method by means of density-scaled $\alpha$-shapes, Int. J. Numer. Methods Eng. 49 (4) (2000) 519-546.

[26] E. Cueto, B. Calvo, M. Doblaré, Modeling three-dimensional piece-wise homogeneous domains using the $\alpha$-shape based natural element method, Int. J. Numer. Methods Eng. 54 (6) (2002) 871-897.

[27] M.A. Martínez, E. Cueto, M. Doblaré, F. Chinesta, A meshless simulation of injection processes involving short fibers molten composites, Int. J. Form. Process. 4 (3) (2001) 217-236.

[28] R. Sibson, A vector identity for the Dirichlet tesselation, Math. Proc. Camb. Phil. Soc. 87 (1980) 151-155.

[29] R. Sibson, Interpreting multivariate data, in: V. Barnett (Ed.), A Brief Description of Natural Neighbor Interpolation, Wiley, New York, 1981, pp. 21-36.

[30] V.V. Belikov, V.D. Ivankov, V.K. Kontorovich, S.A. Korytnik, A. Yu Semenov, The non-Sibsonian interpolation: a new method of interpolation of the values of a function on arbitrary set of points, Comput. Math. Math. Phys. 37 (1) (1997) $9-15$.

[31] N. Sukumar, B. Moran, A. Yu Semenov, V.V. Belikov, Natural neighbor Galerkin methods, Int. J. Numer. Methods Eng. 50 (1) (2001) 1-27.

[32] G.M. Voronoi, Nouvelles Applications des Paramètres Continus à la Théorie des Formes Quadratiques. Deuxième Memoire: Recherches sur les parallélloèdres Primitifs, J. Reine Angew. Math. 134 (1908) 198-287.

[33] B. Delaunay, Sur la Sphre Vide. A la Memoire de Georges Voronoi, Izvestia Akademii Nauk SSSR, Otdelenie Matematicheskii i Estestvennyka Nauk 7 (1934) 793-800.

[34] E. Cueto, J. Cegoñino, B. Calvo, M. Doblaré, On the imposition of essential boundary conditions in natural neighbor Galerkin methods, Numer. Methods Eng., 2002, submitted to publication.

[35] H. Edelsbrunner, D.G. Kirkpatrick, R. Seidel, On the shape of a set of points in the plane, IEEE Trans. Inform. Theory IT-29 (4) (1983) 551-559.

[36] H. Edelsbrunner, E. Mucke, Three-dimensional $\alpha$-shapes, ACM Trans. Graph. 13 (1994) 43-72.

[37] E. Cueto, M.A. Martínez, M. Doblaré, El método de los Elementos Naturales Basado en Formas Alfa en Elasticidad Compresible y Cuasi-Incompresible, Boletín Tcnico del Instituto de Materiales y Modelos Estructurales. Universidad Central de Venezuela, Caracas, Venezuela, vol. 39, no. 3, 2001.

[38] E. Hinton, J.S. Campbell, Local and global smoothing of discontinuous finite element functions using a least squares method, Int. J. Numer. Methods Eng. 8 (1974) 461-480.

[39] D. Gonzalez, E. Cueto, M.A. Martínez, M. Doblar, Numerical integration in natural neighbor Galerkin methods, Int. J. Numer. Methods Eng., 2002, submitted for publication.

[40] E. Cueto, N. Sukumar, B. Calvo, J. Cegoñino, M. Doblare, Overview and recent advances in natural neighbor Galerkin methods, Arch. Comput. Methods Eng. 2003, in press. 